\title{
Pregnancy Promotes Melanoma Metastasis through Enhanced Lymphangiogenesis
}

\author{
Kiarash Khosrotehrani, ${ }^{* \dagger £}$ Sau Nguyen Huu, ${ }^{* \dagger}$ \\ Aurélie Prignon, ${ }^{* \dagger}$ Marie-Françoise Avril, ${ }^{\text {" }}$ \\ Françoise Boitier, ${ }^{\text {" }}$ Michèle Oster, ${ }^{* \dagger}$ \\ Laurent Mortier," Marie-Aleth Richard, ${ }^{* *}$ \\ Eve Maubec, ${ }^{+\dagger}$ Delphine Kerob, ${ }^{\ddagger \neq}$ \\ Sandrine Mansard, $\$$ Charbel Merheb, ${ }^{\text {, }}$ \\ Philippe Moguelet, IIII Dany Nassar, * \\ Sarah Guégan, ${ }^{\star \star \ddagger}$ and Selim Aractingi ${ }^{\star \star \ddagger}$ \\ From the Université Pierre et Marie Curie, University Paris 06,* \\ Paris, France; INSERM UMR_S938, Centre de Recherche Saint \\ Antoine, ${ }^{\dagger}$ Paris, France; the Departments of Dermatology, ${ }^{\ddagger}$ \\ Nuclear Medicine, "वाा and Pathology, III Assistance Publique \\ Hôpitaux de Paris, Hôpital Tenon, Paris, France; the \\ Experimental Dermatology Group, ${ }^{\S}$ University of Queensland \\ Centre for Clinical Research, Brisbane, Australia; the Department \\ of Dermatology," Assistance Publique Hôpitaux de Paris, Hôpital \\ Tarnier, Paris, France; the Department of Dermatology, CHRU \\ Lille, Hôpital Claude Huriez, Lille, France; the Department of \\ Dermatology, ${ }^{* *}$ Assistance Publique-Hôpitaux de Marseille, CHU \\ Sainte-Marguerite, Marseille, France; the Department of \\ Dermatology, ${ }^{\text {t+ }}$ Assistance Publique Hôpitaux de Paris, Hôpital \\ Bichat, Paris, France; the Department of Dermatology, \\ Assistance Publique Hôpitaux de Paris, Hôpital Saint-Louis, \\ Paris, France; and the Department of Dermatology, ${ }^{\$ S}$ \\ Université d'Auvergne Clermont-Ferrand 1, CHU Hôtel-Dieu, \\ Clermont-Ferrand, France
}

The relationships of pregnancy and melanoma have been debatable. Our aim was to assess the influence of gestation on the course of melanoma in a classic murine model of tumor progression and in women. B16 mouse melanoma cells were injected in nonpregnant or pregnant mice on day 5 of gestation. Animals were evaluated for tumor progression, metastases, and survival. Tumor sections were analyzed for lymphatic and blood vessel number and relative surface and expression of angiogenic growth factors. Finally, primary melanomas from pregnant and nonpregnant women, matched for age and tumor thickness, were also considered. Tumor growth, metastasis, and mortality were increased in B16-injected pregnant mice. Tumors displayed an increase in intratumoral lymphangiogenesis during gestation. This increased lym- phatic angiogenesis was not observed in normal skin during gestation, showing its specificity to the tumor. An analysis of melanoma from pregnant and matched nonpregnant women showed a similar increase in lymphatic vessels. Tumors from pregnant mice had increased expression of vascular endothelial growth factor $A$ at the RNA and protein levels. The increased vascular endothelial growth factor A production by melanoma cells could be reproduced in culture using pregnant mouse serum. In conclusion, pregnancy results in increased lymphangiogenesis and subsequent metastasis. Caution should be applied in the management of patients with advanced-stage melanoma during gestation. (AmJ Pathol 2011, 178:1870-1880; DOI: 10.1016/j.ajpath.2010.12.044)

The incidence of melanoma has been increasing sharply, by approximately $10 \%$ a year, in the last decades, resulting in increases of $619 \%$ in annual diagnoses of cutaneous melanoma and $165 \%$ in annual mortality from 1950 to 2000. ${ }^{1}$ Of all cancers in the United States, cutaneous melanoma is the most common among women aged 20 to 29 years. ${ }^{1}$ Because this tumor develops in women of childbearing age, the incidence increase previously described is expected to lead to a parallel increase in melanomas during gestation. Classically, melanoma affected 2.8 to 5 per 100,000 pregnant women, ${ }^{2}$ accounting for approximately eight percent of all malignant neoplasms diagnosed during gestation, ${ }^{3}$ after cervical and

Supported by grants from the Assistance-Publique Hôpitaux de Paris (CRC04026 and CRC06075), the Société Française de Dermatologie, the Association pour la Recherche sur le Cancer, and the Fondation René Touraine (S.N.H.)

K.K., S.G., and S.A. designed the study, analyzed the results, and wrote the manuscript; S.N.H., A.P., and M.O. performed the experiments; M.-F.A., F.B., L.M., M.-A.R., E.M., and D.K. collected and contributed material; and C.M. and P.M. performed the analyses.

Accepted for publication December 30, 2010.

Supplemental material for this article can be found at http://ajp. amjpathol.org or at doi:10.1016/j.ajpath.2010.12.044.

Address reprint requests to Selim Aractingi, M.D., Ph.D., Université Paris 6, 27 rue de Chaligny, 75012 Paris, France. E-mail: selim.aractingi@ gmail.com. 
breast cancer. ${ }^{4}$ However, a recent large survey ${ }^{5}$ from Norway found melanoma as the most frequent neoplasia associated with pregnancy.

The influence of pregnancy on melanoma has been debatable. Pregnancy was initially suspected to worsen the natural course of melanoma, ${ }^{6,7}$ with shorter disease-free survival intervals. ${ }^{8}$ However, later studies $^{9-11}$ did not confirm these findings, at least in earlystage melanoma after careful adjustment of patient outcome on melanoma thickness, Breslow index, a major prognostic factor. However, several of these same studies ${ }^{10,11}$ found that tumor thickness was higher in melanomas during gestation, therefore suggesting an influence of gestation.

In various maternal tumors during pregnancy, fetal chimeric cells may participate in the tumor stroma and contribute to tumor angiogenesis. ${ }^{12-14}$ Given the growing incidence of melanoma in pregnancy and the past controversy on its effect on prognosis, we aimed to determine the influence of gestation on the evolution of maternal melanoma, with a special focus on tumor angiogenesis and lymphangiogenesis. We chose the classic murine B16 model of melanoma metastasis for our study and confirmed our findings in a clinical setting on patients affected with melanoma during and outside gestation. Our results indicate that melanomas during gestation have an increased level of lymphangiogenesis and vascular endothelial growth factor (VEGF) A, possibly explaining the observed increase in metastasis, proliferation, and mortality.

\section{Materials and Methods}

\section{Patients}

Pathology records from different hospitals in France were retrospectively reviewed in accordance with local committee and European Union ethical regulations for patient's information and consent during biomedical research. Women with malignant melanoma or benign nevi were identified and included if the primary tumor had been excised during pregnancy between 1996 and 2008. Histological and clinical parameters, such as Breslow index (tumor thickness), Clark's level, and the presence of ulceration, were obtained. A control group was established; this group was composed of nonpregnant control patients with melanoma, matched for sex, age $( \pm 2$ years), Breslow index $( \pm 0.2 \mathrm{~mm})$, and ulceration status. Pregnant patients with a 2:1 control/patient ratio were included.

\section{Mice}

Wild-type C57BL/6 female mice (aged 8 weeks) were bred to syngenic males. All mice were treated in accordance with the institutional guidelines for the care of experimental animals.

For tumor implantation, 1 million B16-BL6-F10 cells (B16, provided by $\operatorname{Dr} \mathrm{O}$. Lanz, Curie Institute, Paris, France) were inoculated intradermally in the right flanks of all females after 5 days of mating, without knowing the pregnancy status leading to pregnant and nonpregnant mice with tumors.

Similar experiments were performed on a DBA2 background using the S91 murine melanoma cell line (American Type Culture Collection: CCL 53-1). Tumor cell inoculations were performed 10 days before the mating, given the slower progression rate of this tumor cell line.

Both cell lines were tested according to ATCC guidelines, verifying their morphological features, proliferation rate, pigmentation, and engraftment in the appropriate mouse strain to ascertain origin.

\section{Tumor Evaluation}

Tumor growth was followed by a digital caliper. Tumor volume (V) was calculated as follows: $V=a b^{2} \pi / 6$, where $a$ is the longest and $b$ is the shortest of two perpendicular diameters. On day 16 after inoculation, all mice were sacrificed. Pregnancy status was confirmed during sacrifice. Tumors were collected and immediately frozen at $-80^{\circ} \mathrm{C}$. The presence of metastases was systematically evaluated in homolateral and contralateral inguinal and axillary lymph nodes, abdominal lymph nodes, and internal organs, such as the spleen, liver, and lung (macroscopically).

\section{8-Fluorodeoxyglucose Positron Emission Tomography}

In vivo imaging was performed on day 16 after inoculation of tumor cells. After a fasting period of 12 hours, mice were injected i.v. with 6 to $8 \mathrm{MBq}$ of FDG and underwent imaging an hour later, with an exposure time of $15 \mathrm{~min}$ utes under general anesthesia by isoflurane inhalation on a heating plate, maintaining body temperature at $37^{\circ} \mathrm{C}$. Data were analyzed using software (PETView, Philips Medical Systems, Bothell, WA) and interpreted blindly to the pregnancy status by a positron emission tomography (PET) imaging specialist (C.M.). Tumor volumes and intratumoral necrosis were measured on tumor transverse section images, and the percentage of necrotic tissue was calculated. Necrosis was defined as areas inside the tumor that did not uptake FDG. The presence or absence of metastasis was assessed on each image and immediately confirmed after dissection. Tumors of $3 \mathrm{~mm}$ or larger were easily seen on FDG PET images.

\section{Delayed Hypersensitivity Induction}

Oxazolone (Sigma-Aldrich, St Louis, MO) was used to induce delayed hypersensitivity reactions, as previously described. ${ }^{15,16}$ Briefly, on day 10 after mating, pregnant females were shaved and sensitized by a unique topical application on the abdomen of $50 \mu \mathrm{l}$ of oxazolone, two percent solution, in acetone-olive oil (4:1, vol/vol). Six days after sensitization, the right ears were challenged with $10 \mu$ l of oxazolone, two percent solution, in the same vehicle, whereas the left ears were painted with vehicle 
alone. The right ears were rechallenged once per day for 3 days.

\section{Assessment of Inflammation}

For each mouse, ear thickness (right and left) was assessed daily during the ear challenge and before sacrifice using a dial caliper (precision, $0.01 \mathrm{~mm}$ ) (Mitutoyo, Kanagawa, Japan). In addition, a semiquantitative microscopic assessment (standard H\&E staining) of the level of skin inflammation was performed blindly. This assessment evaluated the degree of the edema, the inflammatory cell infiltration, and the epidermal lesions; each item was graded from absent to strong (score, 0 to 3 ).

\section{In Vivo Laser Doppler Perfusion Imaging}

To provide functional evidence for inflammation and gestation-induced changes in skin vascularization, laser Doppler perfusion imaging experiments were performed on both ears, as previously described. ${ }^{14}$ Numeric data and images were collected on day 20 after mating ( 1 day after the last oxazolone application and before sacrifice), under general anesthesia by ketamine-xylazine injection. Mice were placed on a heating plate at $37^{\circ} \mathrm{C}$ to minimize temperature variation. To account for variables, including ambient light and temperature, calculated perfusion was expressed in arbitrary units and was compared between pregnant and nonpregnant animals on inflamed and noninflamed ear skin.

\section{Immunostaining}

Immunofluorescence staining was performed on human and animal sections, as described. Briefly, after permeabilization (Triton X-100), sections were blocked using $20 \%$ normal goat serum (DakoCytomation; Dako, Trappes, France). The primary antibodies used were the monoclonal mouse anti-human CD34 (1:40; DakoCytomation), the rabbit polyclonal antibody anti-mouse Lyve-1 (1:800; Abcam, Cambridge Science Park, UK), the rabbit anti-Prox1 (AngioBio, DelMar, CA), and purified anti-mouse CD31/P endothelial cell adhesion model 1 (1:40; BD Pharmingen, San Jose, CA). The secondary antibodies were the goat anti-rabbit IgG labeled with fluorescein isothiocyanate, goat anti-rabbit IgG labeled with Texas Red, goat anti-rat IgG labeled with Texas Red (1:100; Jackson Immunoresearch, West Grove, PA), and goat anti-mouse IgG labeled with Texas Red (1:100; Jackson Immunoresearch). Nuclei were counterstained with $0.3 \mu \mathrm{g} / \mathrm{mL}$ DAPI. Slides were then observed under a fluorescence microscope (Leica, Deerfield, IL) with a digital camera (QImaging; Media Cybernetics, Silver Spring, MD). Immunoperoxidase staining for anti-D2-40 (1:10; Biovalley, Marne-LaVallée, France) was performed on human paraffin-embedded sections using a system (Envision + Dual Link Peroxidase system; DakoCytomation).

\section{Morphometric Analysis of Vessel Density}

Paraffin-embedded sections of human melanoma were immunostained for D2-40 or CD34. Frozen sections of B16-BL6 melanomas from pregnant and nonpregnant mice were double stained for Lyve-1 and CD31. To account for the variability of the staining procedure, cases and matched controls were always stained and analyzed on the same day. Sections were then examined with a fluorescent microscope (Leica), and digital images were captured using a digital camera (SPOT; Media Cybernetics, Silver Spring, MD). For each tumor section, three fields within and three fields surrounding the tumor with the highest vascular density (hot spots) were evaluated at $\times 100$ magnification. The peritumoral region was defined within an area of $100 \mu \mathrm{m}$ from the tumor borders that were determined on serial sections using DAPI and H\&E stains.

Morphometric analyses of lymphatic and blood vessels were performed using computer-assisted morphometric vessel analysis software (Mercator; Explora Nova, LaRochelle, France) to determine the number of vessels per millimeter squared, the average size of vessels, and the relative area occupied by vessels, as previously described. ${ }^{17}$ This analysis was performed blinded to pregnancy status.

\section{Real-Time Quantitative RT-PCR}

Total RNA was extracted from tumors using a mini kit (RNeasy; Qiagen, Courtaboeuf, France), according to the manufacturer's instructions. cDNA was synthesized from the purified RNA using a high-capacity cDNA archive kit (Applied Biosystems, Courtaboeuf, France). Real-time quantitative RT-PCR was performed on a system (ABI PRISM 7300 Sequence Detection System; Applied Biosystems) using SYBR Green PCR master mix (Applied Biosystems). The PCR reaction was performed in a 25- $\mu$ l volume containing $2.5 \mu \mathrm{l}$ of CDNA, $\times 1$ SYBR Green PCR master $\mathrm{mix}$, and $800 \mathrm{nmol} / \mathrm{L}$ of the forward and reverse primers. Primers were synthesized according to the method of Thijssen et al. ${ }^{18}$ The PCR profile was as follows: 10 minutes at $95^{\circ} \mathrm{C}$, followed by 40 cycles of 15 seconds at $95^{\circ} \mathrm{C}$, and 1 minute at $60^{\circ} \mathrm{C}$. After PCR, a melting curve was constructed by increasing the temperature from $65^{\circ} \mathrm{C}$ to $95^{\circ} \mathrm{C}$, with a transition rate of $0^{\circ} \mathrm{C} / \mathrm{s}$ to $1^{\circ} \mathrm{C} / \mathrm{s}$ to verify the specificity of the desired PCR products and the absence of primers-dimers. The mean target gene mRNA expression level was calculated using the $2^{-\Delta \Delta \mathrm{CT}}$ method for each gene after reporting expression of $\beta$-actin or cyclophilin.

\section{Enzyme-Linked Immunosorbent Assay}

Total proteins were extracted from tumors using a kit (QProteome Mammalian Protein Prep kit; Qiagen). The VEGF and placenta-like growth factor (PIGF) levels were also determined using a kit (Quantikine VEGF or PIGF2 ELISA kit, respectively; R\&D Systems Europe RD, Minneapolis, MN). The VEGF-C levels were quantified using a detection kit (Bender MedSystems GmbH, Vienna, Aus- 
Table 1. Characteristics of B16-BL6 Melanomas in Mice

\begin{tabular}{|c|c|c|c|}
\hline Characteristics & Pregnant, $n=24(\mathrm{Cl} 95 \%)$ & Nonpregnant, $n=28$ (Cl 95\%) & $P$ value \\
\hline \multicolumn{4}{|l|}{ Volume $\left(\mathrm{cm}^{3}\right)$} \\
\hline Day 10 & $0.89(0.30-2.87)$ & $0.48(0.09-1.50)$ & $0.015^{*}$ \\
\hline Day 12 & $1.66(0.41-4.91)$ & $1.22(0.51-3.45)$ & $0.087^{*}$ \\
\hline Day 14 & $3.59(0.79-6.81)$ & $2.83(1.16-6.70)$ & $0.141^{*}$ \\
\hline Metastases & 9 & 2 & $0.015^{\dagger}$ \\
\hline Tumor weight on day 16 (g) & $4.00(0.62-8.78)$ & $3.86(1.69-7.07)$ & $0.412^{*}$ \\
\hline \multicolumn{4}{|l|}{ FDG PET on day 16} \\
\hline No. & 16 & 21 & \\
\hline Volume $\left(\mathrm{cm}^{3}\right)$ & $4.85(0.64-8.55)$ & $3.53(1.02-6.72)$ & $0.046^{*}$ \\
\hline Necrotic tissue (\%) & 12.6 & 18.3 & $0.065^{*}$ \\
\hline
\end{tabular}

Data are given as the mean unless otherwise indicates.

*Statistical analyses were performed using the Student's t-test.

tStatistical analyses were performed using the Fisher's exact test.

tria). The VEGF kit specifically measures rodent VEGF164 and VEGF120 variants, although the PIGF2 kit detects mouse PIGF. The detection limit for both proteins was 15 $\mathrm{pg} / \mathrm{mL}$. Concentrations were normalized relative to cellular protein concentration by Bradford protein assay in tumor samples.

\section{Statistical Analysis}

Categorical variables were compared using Fisher's exact test. Continuous variables were compared using Student's t-test or analysis of variance. In the absence of normal distribution, the Mann-Whitney-Wilcoxon test was used for these comparisons. Kaplan-Mayer analysis was used for survival comparisons over time. $P<0.05$ was considered significant.

\section{Results}

\section{Tumor Growth and Metastasis Are Accelerated during Pregnancy}

A million B16 cells were injected intradermally in the right flanks of 24 pregnant mice on day 5 of mating and in 28 nonpregnant female controls. Tumors developed in all mice and were measured on days 10 to 16 after injection. The tumor volume was significantly higher in pregnant mice on days 10 and 16 (Table 1, Figure 1A).

In addition, on day 16, the tumor volume was calculated using FDG PET images and was significantly increased in pregnant mice $(P<0.05)$. On sacrifice, the tumor weights of pregnant mice appeared higher as well (nonsignificant) (Table 1). In a separate group of mice, we observed a significant decrease in survival in pregnant mice with melanoma compared with nonpregnant controls $(P=0.0158)$ (Figure $1 \mathrm{~B})$. On day 21, 14 of 22 mice were dead in the pregnant group compared with 4 of 16 nonpregnant controls.

Next, we used the FDG intake as a marker of living areas within the tumor as opposed to necrotic areas. By using FDG PET, the relative volume of necrosis in melanomas in pregnant mice was lower than in nonpregnant controls, despite their larger volume, suggesting important modifications in vascularization (Figure 1, C and D; Table 1; $P=0.065$ ).
The presence of metastasis was analyzed by FDG PET imaging (Figure 1, E and $F$ ) and confirmed on dissection in all mice. On day 16, 9 (38\%) of 24 pregnant mice had metastasis, mostly in lymph nodes, compared with only $2(7 \%)$ of 28 nonpregnant mice $(P=0.015)$. Three pregnant mice had multiple metastases to almost all organs; however, in most cases with metastases, tumors had invaded homolateral inguinal nodes and had spread to the abdominal cavity via the iliac lymphatic chains.

\section{Angiogenesis, Lymphangiogenesis, and Cell Proliferation Are Stimulated in Melanomas during Gestation}

Pregnant $(n=6)$ and nonpregnant $(n=10)$ mice with tumors received bromodeoxyuridine for 3 days before sacrifice on day 16 . The bromodeoxyuridine incorporation was then examined on an average of 15 tumor sections for each individual mouse. Melanomas from pregnant mice had more bromodeoxyuridine-labeled nuclei than those from nonpregnant matched controls (50\% versus $38 \% ; P=0.04$ )

The B16 melanoma sections from 23 nonpregnant and 25 pregnant mice were stained with a lymphatic marker (Lyve-1) and a vascular marker (CD31) (Figure 2). In all tumors from both groups, we found a ring of lymphatic vessels immediately surrounding the tumors without any other type of blood vessel. In the periphery of this ring (and inside the tumors), CD $31^{+}$blood vessels were observed. Individual cells stained with Lyve-1 (potential Lyve $-1^{+}$macrophages) were rare and were not included in our surface and density analysis. This staining pattern was also confirmed using another lymphatic-specific antibody, Prox-1 (see Supplemental Figure S1 at http://ajp. amjpathol.org). Indeed, on serial sections of B16 tumors, double staining using CD31 and Lyve-1 or CD31 and Prox-1 produced close results for surface of area stained and vessels identified.

Within tumors from pregnant mice, the relative area stained by Lyve-1-positive lymphatic vessels was much higher compared with the area from nonpregnant mice (5.3\% versus $1.6 \% ; P=0.0016$ ) (Figure 2 and Figure 3 ). The level of lymphangiogenesis in the peritumoral area was comparable in pregnant and nonpregnant animals. 

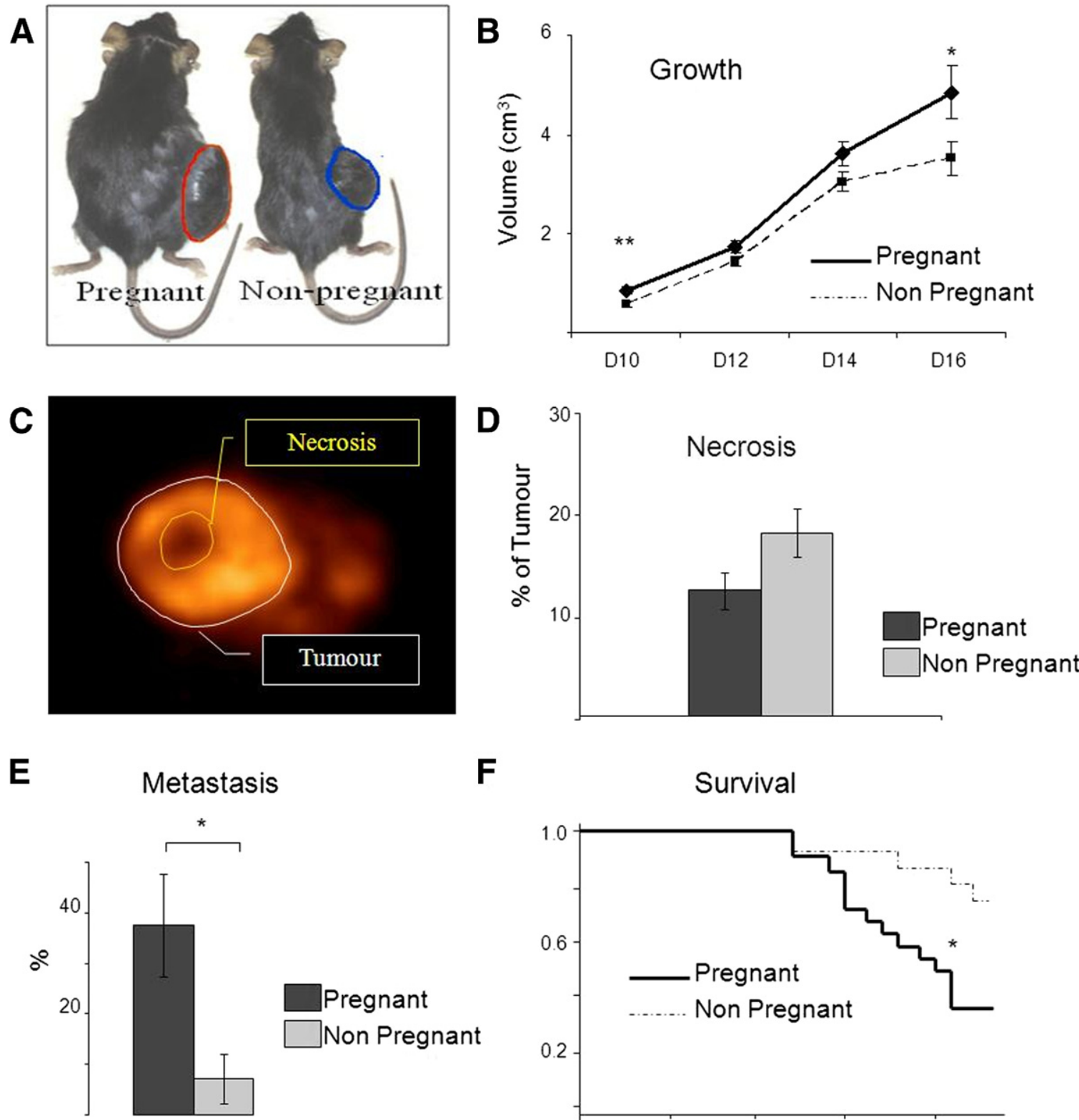

$\mathbf{F}$

Survival

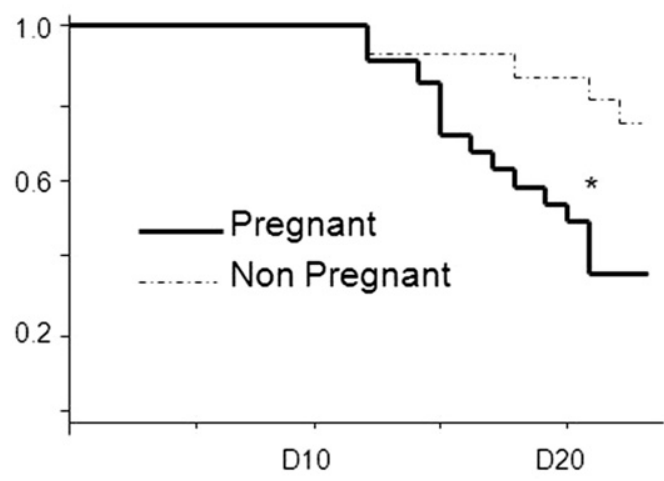

Figure 1. Pregnancy enhances B16 tumor growth, metastasis, and mortality. A: The B16 melanoma cell line was injected in nonpregnant and pregnant mice on day 5 of gestation. B: Tumor volume measured at different points. D indicates day. Volume was also measured using FDG PET on D16. Data are expressed as mean \pm SE. ${ }^{*} P<0.05 ;{ }^{* *} P<0.01$ (Student's $t$-test). C: FDG PET imaging showing necrotic areas in the tumor. D: The percentage of necrotic volume within the tumor on D16 after injection was calculated based on FDG PET imaging in 17 pregnant and 20 nonpregnant mice. Data are expressed as mean \pm SE. $P=0.065$, Student's $t$-test. E: Frequency of metastasis of B16-BL6 melanoma on D16. Data are given as mean \pm SE, ${ }^{*} P<0.05$, Fischer's exact test. F: Survival was better in nonpregnant $(n=16)$ compared with pregnant $(n=22)$ mice by Kaplan-Meier analysis $(P=0.0158)$.

When examining the whole tumor combining intratumoral and peritumoral areas, there was still a significant increase in relative area occupied by lymphatic vessels and in the size of lymphatic vessels during pregnancy $(P=0.029$ and $P=0.049$, respectively; Figure 3).

There was also a significant increase in the CD31 vessel numbers during pregnancy in the tumors and the tumor periphery and an overall increase in CD31 ${ }^{+}$vessel areas in pregnant mice (Figure 3).

To validate our results, we repeated these experiments using another murine melanoma cell line and genetic background: the S91 nonmetastatic melanoma cell line in syngeneic DBA2 mice (Figure 4). ${ }^{19}$ Because tumor growth in this cell line is much slower, the S91 line had to be injected 10 days before mating. Tumor size was not significantly increased in the pregnant group $(n=7)$ on day 28 after injection compared with the nonpregnant group $(n=17)$, possibly as a result of slow tumor progression in this particular cell line (Figure 4). We did not analyze metastases and survival in this line, which has been described as low/nonmetastatic. By analyzing vascular structures within the tumor, we again found an increase in lymphangiogenesis in the pregnant group for relative surface area $(1.1 \%$ 

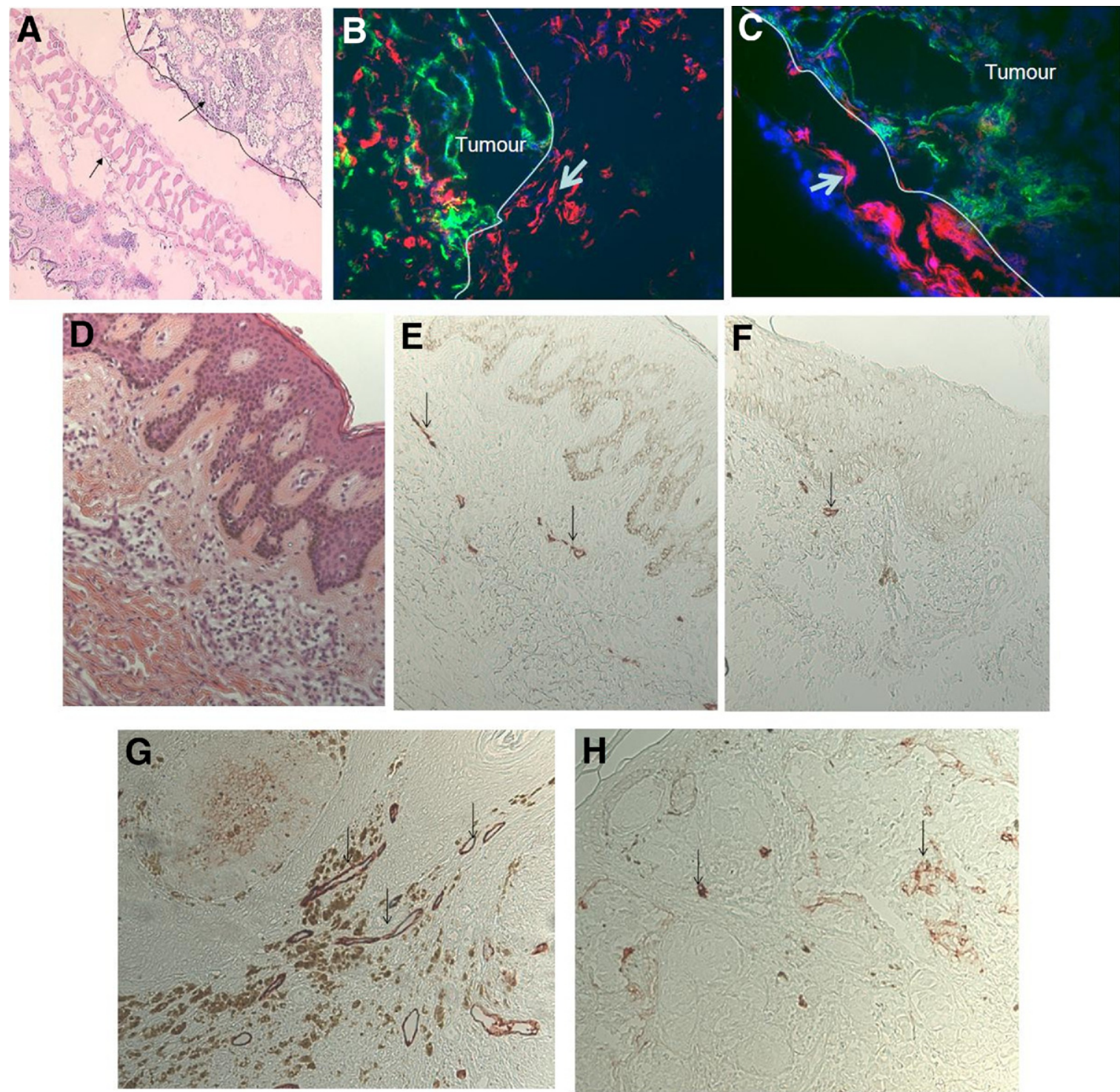

Figure 2. Labeling of intratumoral and peritumoral vessels in melanomas in pregnant and nonpregnant mice and women. A-C: Staining of mouse melanomas. A: H\&E staining of B16 tumors in mice. A layer of vessels (arrow) surrounds the tumor limits (black line, $\times 100$ magnification). B and C: Immunofluorescence staining of B16 melanoma for lymphatic marker Lyve-1 (red) and panvascular marker CD31 (green) reveals prominent lymphatic vessel density (arrow) in the tumor periphery. There is a marked increase in intratumoral lymphatics in tumors from pregnant (B) compared with nonpregnant (C) animals. D-H: Staining on human primary melanomas. D: H\&E-stained section of a human melanoma (Breslow index, 0.35 mm) during pregnancy. E: D2-40 lymphatic staining. Level of lymphatics in the vicinity of the primary tumor (arrows) is increased in pregnant (E) compared with nonpregnant matched control (F). This increased lymphangiogenesis was true in stage II melanomas of pregnant $(\mathbf{G})$ compared with nonpregnant matched control (H) (Breslow index, $1.6 \mathrm{~mm})$.

versus $0.2 \% ; P=0.01$; Figure 4$)$ and density of lymphatic vessels $\left(299 / \mathrm{mm}^{2}\right.$ versus $\left.54 / \mathrm{mm}^{2} ; P=0.05\right)$. There was also an increase in the density of $\mathrm{CD} 31^{+}$ vessels within the tumor $(P=0.05)$. Therefore, in two different cell lines and genetic backgrounds, pregnancy induced an increase in lymphangiogenesis in melanomas.

\section{Lymphangiogenesis Is Increased in Pregnant Women with Melanoma}

Next, we sought to confirm these animal studies in samples obtained from women with melanoma. We analyzed a group of primary melanomas from pregnant compared with nonpregnant women, matched for age, tumor thickness (Breslow index), and presence or absence of ulceration (Table 2). We used CD34 as a marker of blood endothelial cells and D2-40 as a marker of lymphatic vessels (Figure 2). Computer-assisted morphometric analysis showed that the number of intratumoral lymphatic vessels was significantly increased in melanomas from pregnant compared with nonpregnant women [117.4 vessels $/ \mathrm{mm}^{2}(n=14)$ versus 54.8 vessels $/ \mathrm{mm}^{2}$ $(n=26) ; P=0.02 ;$ Table 2, Figure 5]. Furthermore, the relative intratumoral lymphatic area was two times higher in melanomas from pregnant women compared with nonpregnant controls (3.9\% versus $1.5 \% ; P=0.0338)$. In the periphery of tumors, the number of lymphatic vessels per 

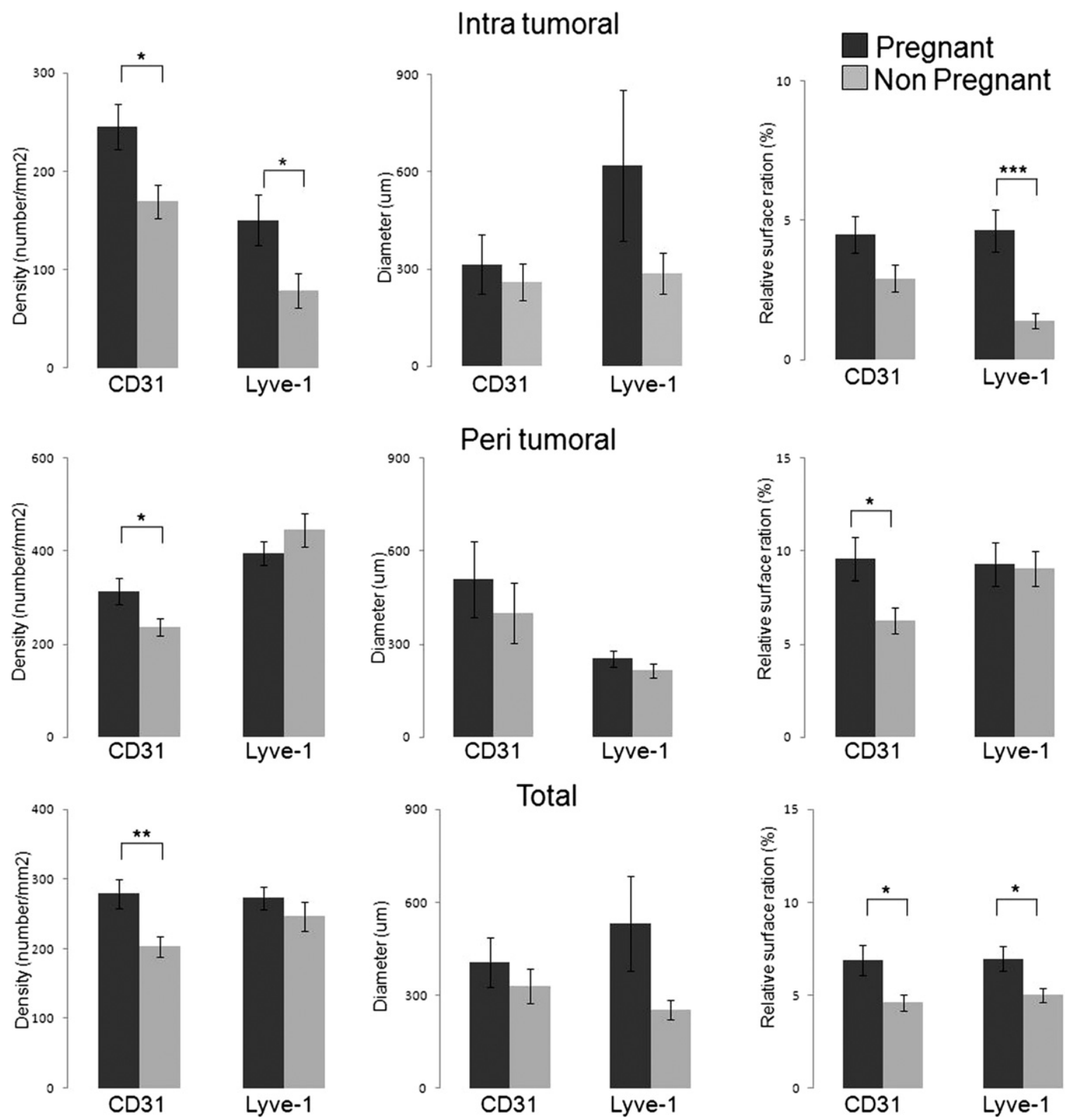

Figure 3. The tumor vessel area is increased in mouse B16-BL6 melanoma during pregnancy. Quantitative analyses were performed after staining of CD31- for blood vessels and Lyve-1 for lymphatic vessels. By using automated morphometric analyses, the following parameters were compared: vascular density, size of vessels, and ratio of relative area occupied by vessels. Results are expressed as mean \pm SE. The Student's $t$-test was used. ${ }^{*} P<0.05,{ }^{* * *} P<0.01$, and ${ }^{* * * *} P<0.001$.

millimeter squared and the relative area of lymphatic vessels were not higher in melanomas of pregnant women, despite a trend. In contrast, pregnancy did not significantly modify the level of blood angiogenesis identified by CD34 staining.

\section{Normal Skin and Delayed Hypersensitivity Reaction during Pregnancy Do Not Result in Increased Blood Flow or Angiogenesis}

To assess whether the observed differences in lymphangiogenesis were specific to tumors or whether they would be observed in different situations during gestation, we analyzed the influence of gestation on the level of angio- genesis in maternal skin at steady state or during contact hypersensitivity. In vivo laser Doppler perfusion imaging was performed on delayed hypersensitivity inflamed and control ears of 42 mice (16 pregnant and 26 nonpregnant) to assess changes in blood perfusion induced by pregnancy and inflammation (see Supplemental Figure S2 at http://ajp.amjpathol.org). Blood perfusion was increased in inflamed ear skin compared with normal skin (inflamed/noninflamed skin ratio, 1.31; $P=0.004$ ). There was no difference observed in relation to gestation: the ratios of pregnant/nonpregnant perfusion in normal and inflamed skin were 1.05 and 1.10, respectively (nonsignificant). The level of inflammation was not different between pregnant and nonpregnant mice, either clinically 


\section{D1 Tumour growth D11 D28}
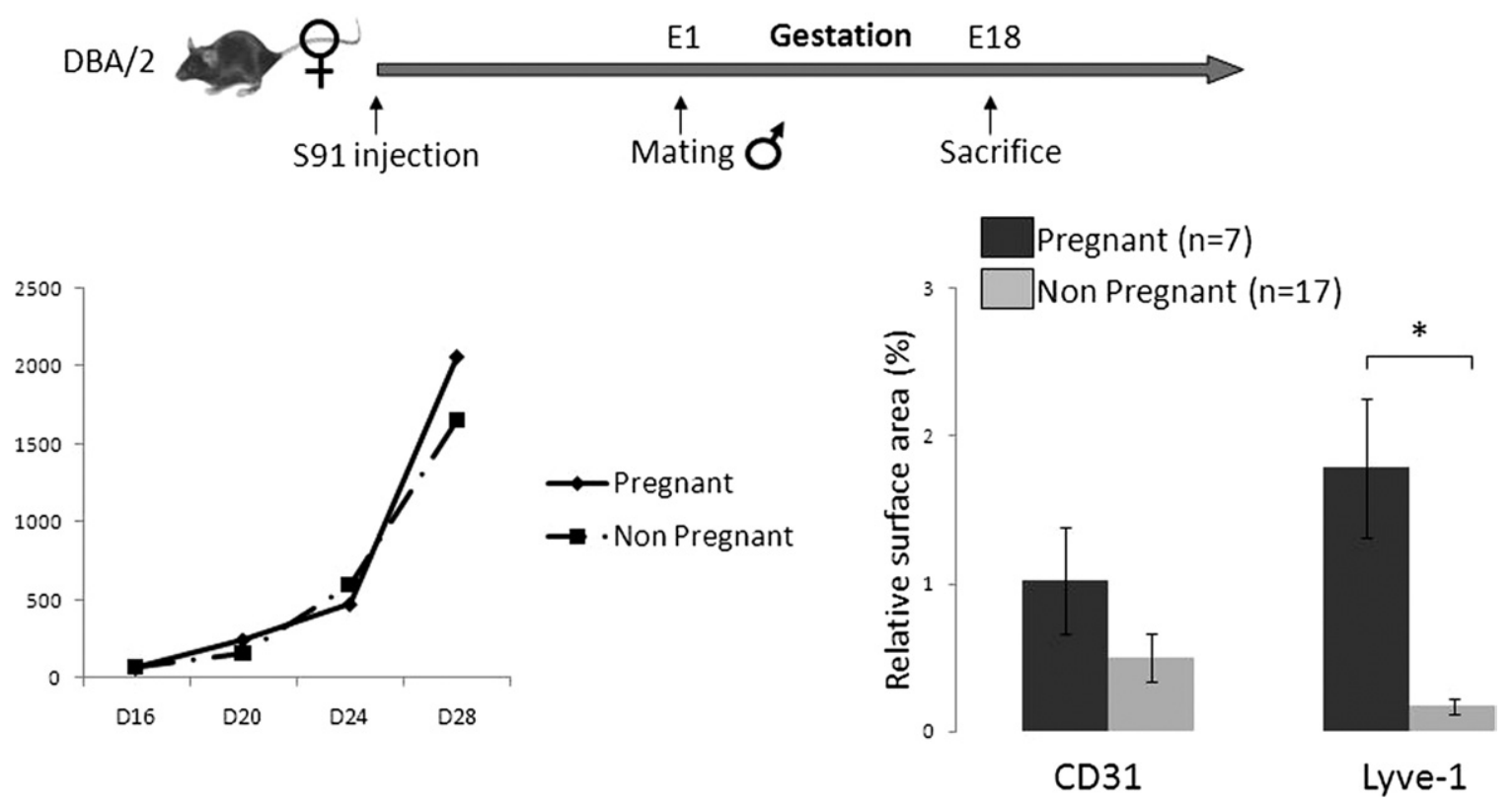

Figure 4. An S91 melanoma cell line-derived tumor displayed increased lymphangiogenesis during pregnancy. Left: The tumor volume (in cubic millimeters) was not different between pregnant and nonpregnant mice. Right: Lymphatic and blood vessel angiogenesis was also evaluated on tumor sections from pregnant and nonpregnant mice using computerized automated morphometric analysis. The total lymphatic vessel relative surface area within tumors was increased during gestation $(* P=0.012)$.

or histologically (data not shown). Similarly, morphometric analysis using CD31 and Lyve-1 on 35 pregnant and 48 nonpregnant mice did not reveal any changes as the result of pregnancy in normal or inflamed skin. Inflamed skin had expectedly higher levels of blood and lymphatic vessel areas $(P<0.001)$. Altogether, these results suggest that the increased lymphangiogenesis observed during pregnancy in melanoma is due to angiogenic factors produced by the tumor and not circulating factors during pregnancy.

Table 2. Vascular Lymphatic Morphometry in Melanomas from Women

\begin{tabular}{|c|c|c|c|}
\hline Variable & $\begin{array}{l}\text { Pregnant } \\
(n=14)\end{array}$ & $\begin{array}{l}\text { Nonpregnant } \\
\quad(n=26)\end{array}$ & $\begin{array}{c}P \\
\text { value }\end{array}$ \\
\hline Age (years) (Cl 95\%) & $31(27-36)$ & $31(24-37)$ & 0.75 \\
\hline \multicolumn{4}{|l|}{$\begin{array}{l}\text { Breslow index, mm } \\
\text { (No. of patients) }\end{array}$} \\
\hline$<1.5$ & 9 & 19 & NA \\
\hline$\geq 1.5$ & 5 & 7 & NA \\
\hline Mean & 1.4 & 1.3 & 0.74 \\
\hline \multicolumn{4}{|l|}{$\begin{array}{l}\text { Lymphatic vascular } \\
\text { area (\%) }\end{array}$} \\
\hline Intratumoral & 3.9 & 1.5 & 0.034 \\
\hline Peritumoral & 1.8 & 1 & 0.10 \\
\hline \multicolumn{4}{|l|}{$\begin{array}{l}\text { Lymphatic vascular } \\
\text { density }\left(\mathrm{NBR} / \mathrm{mm}^{2}\right)\end{array}$} \\
\hline Intratumoral & 117 & 55 & 0.02 \\
\hline Peritumoral & 31 & 19 & 0.09 \\
\hline
\end{tabular}

Data are given as the mean unless otherwise indicated. Statistical analyses were performed using the Student's $t$-test.

NBR, number of vessels.

\section{Expression of VEGF-A and PIGF, but Not VEGF-C, Is Increased in Tumors during Gestation}

Finally, we examined the expression of candidate molecules important in angiogenesis and lymphangiogenesis in tumors during and outside gestation. A total of 28 pregnant and 23 nonpregnant mice from two independent experiments were assayed. Real-time quantitative amplification of cDNA sequences reported to $\beta$-actin showed a significant increase in RNA expression of VEGF-A, VEGF-B, PIGF, Neuropilin1, VEGF receptor (VEGFR) 2, and VEGFR1 (Figure 6). The expression of VEGF-A RNA was an average of four times higher during gestation $(P=0.01)$. The levels of VEGF-C and VEGFR3, classically implicated in lymphangiogenesis, were not increased in tumors during pregnancy. Similar results were observed when cyclophilin was used as a housekeeping gene (data not shown).

We then evaluated tumor lysates for the levels of VEGF-A, VEGF-C, and PIGF proteins. By using an enzyme-linked immunosorbent assay, tumors from pregnant mice displayed significantly higher levels of VEGF-A compared with those from nonpregnant counterparts ( $n=20$ per group). However, PIGF and, as expected, VEGF-C levels were not different between both groups (Figure 6). This pattern of expression of VEGF-A and PIGF, but not VEGF-C, could be reproduced in vitro by culturing B16 cells in the presence of pregnant mouse serum (see Supplemental Figure S3 at http://ajp. amjpathol.org). 

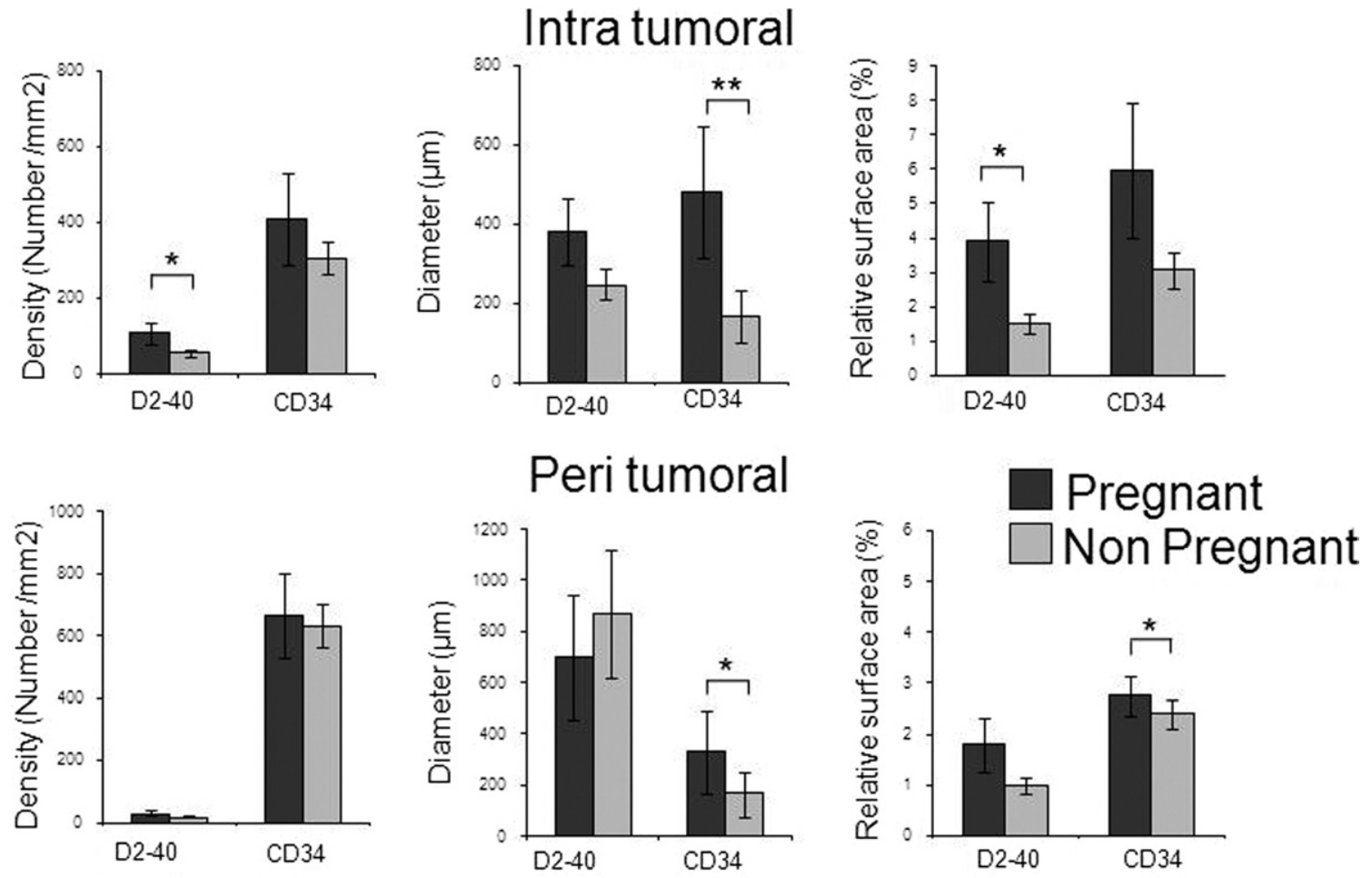

\section{Total}
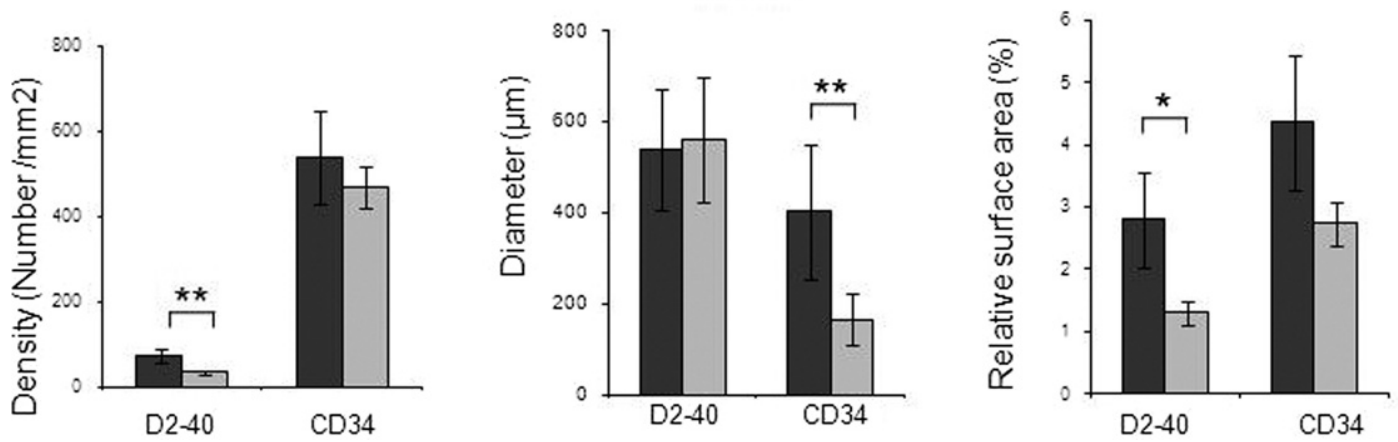

Figure 5. Tumor lymphatic vessel area is increased in human melanomas during pregnancy. Quantitative analyses were performed after immunostaining of CD34- for blood vessels and D2-40 for lymphatic vessels. By using automated morphometric analyses, the following parameters were compared: vascular density, size of vessels, and ratio of relative area occupied by vessels. Results are expressed as mean \pm SE. The Student's $t$-test was used. ${ }^{*} P<0.05$, ${ }^{* *} P<0.01$.

\section{Discussion}

The relationships of pregnancy and melanoma have been debatable for many years. In the present study, we directly assessed the influence of gestation on melanoma. We demonstrated that, during gestation, melanomas grew more rapidly and had increased metastasis and mortality in a model of tumor metastasis. Such behavior could be linked to stimulated lymphangiogenesis during gestation, as observed in two mouse models and in a human case-control series.

Once primary melanoma develops, the critical step that influences the natural history of the disease is lymphatic metastasis. Accordingly, lymph node invasion, as determined by a positive sentinel lymph node biopsy result, is a major determinant for the prognosis of melanoma. ${ }^{20}$ In addition, several studies have demonstrated that the development of macroscopic ${ }^{21,22}$ or microscopic $^{23-25}$ lymph node invasion was dependent on the level of lymphangiogenesis at the site of the primary tumor. In agreement, increased lymphangiogenesis was the best independent predictive factor for sentinel lymph node invasion in cohort analyses, with a hazard ratio of 5.5.25 Tumor lymphangiogenesis has also been an important prognostic factor in melanoma, independently of the Breslow index. ${ }^{26}$ Finally, primary tumors induce new lymphatic vessel growth in draining lymph nodes before metastasis as a potential way to facilitate tumor spread. ${ }^{21}$ Evaluation of blood vessel angiogenesis was performed with a single CD31 marker and could have been modified by CD31+ lymphatic vessels. Overall, our data did not support an increase in blood vessel angiogenesis because this was not observed in the S91 model or the human samples. 

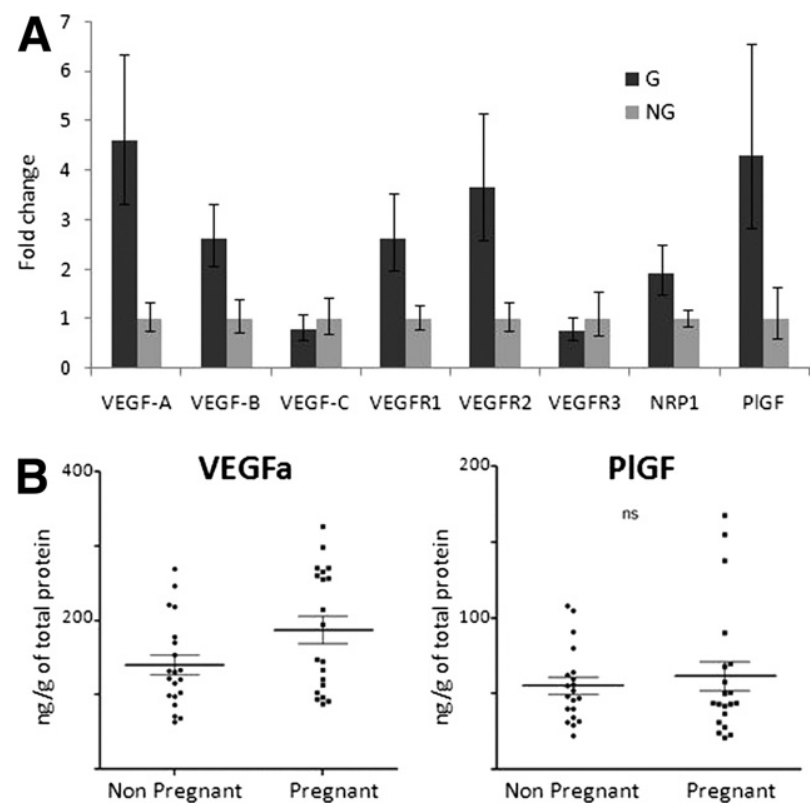

Figure 6. The B16 murine melanomas during gestation express higher levels of VEGF-A. A: Real-time quantitative RT-PCR evaluation of angiogenic factors expressed by tumors from pregnant and nonpregnant mice. Results were combined from two independent experiments. Comparisons reached significance for VEGF-A $(P=0.001)$ and VEGF-B $(P=0.02)$, VEGFR1 $(P=0.02)$ and VEGFR2 $(P=0.006)$, Neuropilin1 $(P=0.04)$, and PlGF $(P=0.03)$ using the Student's $t$-test. G, gestant; NG, non gestant; NRP, neuropilin. B: Evaluation of VEGF-A and PlGF protein expression levels in tumors from pregnant and nonpregnant mice using an enzyme-linked immunosorbent assay. After protein extraction from tumor samples, total protein levels were quantified. Results are expressed as quantity of VEGF-A or PlGF reported to total proteins within the sample. Each dot represents an individual mouse. The difference between pregnant and nonpregnant groups was significant for VEGF-A $(P=0.04)$.

Altogether, these data indicate the role of lymphangiogenesis in the metastatic spread of melanoma. Herein, in two independent mouse models, we demonstrated an increase in lymphangiogenesis in tumors during pregnancy compared with controls (not pregnant). S91 is a slow-growing cell line with limited metastatic potential. Therefore, it was not evaluated for progression, survival, and metastasis. ${ }^{19} \mathrm{~B} 16$ is a murine melanoma cell line largely studied as a valid tool for the evaluation of lymph node invasion. Indeed, the incidence of regional lymph node metastases correlates with increasing primary tumor size. ${ }^{27}$ The B16 cells attach to lymph node reticular fibers through $\beta 1$ integrin receptor complexes, ${ }^{28}$ and there are undetectable proliferating (occult) or nonproliferating (dormant) micrometastases, as in human disease. ${ }^{29}$ Therefore, although B16 differs from human primary tumor development in the epidermis, well reproduced in BRAF, PINK4, or PTEN genetically modified animals, ${ }^{30}$ it mimics well the metastatic course of the human disease. Indeed, the abnormalities in lymphangiogenesis that we found in B16-injected mice were reproduced in patients, strengthening its validity as a model for this critical step. The lymphatic vessel number and relative area were twice as high in women affected with melanoma during pregnancy compared with nonpregnant controls matched for age, Breslow index, and ulceration status. We do not have any indication about the functionality of these lymphatic ves- sels because many of these newly formed vessels may not contribute to lymphatic flow. ${ }^{31}$ However, these findings corroborate our observations in mice, suggesting that, with an equal Breslow index, pregnant women are at increased risk of melanoma lymphatic spread. Interestingly, in both models, lymphatic vessel angiogenesis was increased within the tumor, although blood vessel angiogenesis was increased in the periphery of the tumor. These exact similarities further suggest a common underlying mechanism.

Our results exclude a role for circulating levels of angiogenic factors during pregnancy in promoting increased lymphangiogenesis because this observation was specific to skin tumors and did not occur on normal or inflamed skin. The expression of angiogenic factors, such as VEGF-C or VEGF-D, by tumor cells has played a key role in the development of lymphatic vessels through VEGFR3 binding. ${ }^{25,32,33}$ Vascular endothelial growth factor $A$ and PIGF have also previously promoted lymphangiogenesis. In transgenic animals overexpressing VEGF-A or PIGF in the skin, increased vessel formation, including lymphatics, occurred within normal or inflammatory skin and tumors. ${ }^{21,34}$ In the latter, this resulted in an increased rate of metastases. Finally, proliferation of lymphatic endothelial cells was triggered by VEGF-A through the activity of endothelial-specific molecule $1 .{ }^{35}$ However, evaluation of blood vessel angiogenesis was performed with a single CD31 marker and could have been modified by CD31 ${ }^{+}$ lymphatic vessels.

Early studies reported the influence of pregnancy on melanoma dissemination. Indeed, the disease-free interval from 58 women with melanoma during pregnancy was shorter when compared with 2938 matched controls. ${ }^{8}$ Slingluff et $\mathrm{al}^{36}$ found an increased incidence of lymph node metastases and a significantly shorter disease-free interval in pregnant patients compared with controls, despite accounting for the Breslow index in multivariate analysis. Again, in a recent cohort study, Stensheim et al ${ }^{5}$ reported a significant increase of cause-specific death in patients diagnosed as having melanoma during gestation. Other large studies ${ }^{9-11}$ demonstrated that pregnancy did not modify the course of melanoma. However, most patients included had thin stage I and II melanomas only. The animal experiments conducted in our study mimic later stages of melanoma. Therefore, our current results do not contradict the previously described studies. Finally, in several well-conducted cohort studies, ${ }^{10,11}$ the Breslow index was higher in melanomas that occurred during gestation. The proposed explanation for this finding was that women sought advice later during pregnancy for lesions suggestive of melanoma. An alternative explanation to the increase in melanoma thickness brought by our results would be a role of pregnancy through enhanced angiogenesis.

In conclusion, we show that, in a classic animal model of spread and metastasis, melanomas during gestation grow faster, invade lymph nodes more frequently, and result in reduced survival. This appears to relate to enhanced lymphangiogenesis during gestation. More important, we confirmed these findings in a clinical setting, suggesting to be cautious in melanomas during gesta- 
tion. Further studies are needed to definitely identify the implicated factors that drive this increased lymphangiogenesis during pregnancy.

\section{Acknowledgment}

We thank Professor Francoise Montravers for allowing us access to FDG scintigraphy at the Tenon Hospital small animal PET imaging center.

\section{References}

1. Tsao H, Atkins MB, Sober AJ: Management of cutaneous melanoma. N Engl J Med 2004, 351:998-1012

2. Dillman RO, Vandermolen LA, Barth NM, Bransford KJ: Malignant melanoma and pregnancy ten questions. West J Med 1996, 164:156-161

3. Wong JH, Sterns EE, Kopald KH, Nizze JA, Morton DL: Prognostic significance of pregnancy in stage I melanoma. Arch Surg 1989, 124:1227-1230

4. Lishner M: Cancer in pregnancy. Ann Oncol 2003, 14(Suppl 3): iii31-iii36

5. Stensheim H, Moller B, van Dijk T, Fossa SD: Cause-specific survival for women diagnosed with cancer during pregnancy or lactation: a registry-based cohort study. J Clin Oncol 2009, 27:45-51

6. Pack GT, Scharnagel IM: The prognosis for malignant melanoma in the pregnant woman. Cancer 1951, 4:324-334

7. Houghton AN, Flannery J, Viola MV: Malignant melanoma of the skin occurring during pregnancy. Cancer 1981, 48:407-410

8. Reintgen DS, McCarty KS Jr, Vollmer R, Cox E, Seigler HF: Malignant melanoma and pregnancy. Cancer 1985, 55:1340-1344

9. O'Meara AT, Cress R, Xing G, Danielsen B, Smith LH: Malignant melanoma in pregnancy: a population-based evaluation. Cancer 2005, 103:1217-1226

10. MacKie RM, Bufalino R, Morabito A, Sutherland C, Cascinelli N: Lack of effect of pregnancy on outcome of melanoma: for the World Health Organisation Melanoma Programme. Lancet 1991, 337:653-655

11. Travers RL, Sober AJ, Berwick M, Mihm MC Jr, Barnhill RL, Duncan LM: Increased thickness of pregnancy-associated melanoma. $\mathrm{Br} J$ Dermatol 1995, 132:876-883

12. Nguyen Huu S, Oster M, Avril MF, Boitier F, Mortier L, Richard MA, Kerob D, Maubec E, Souteyrand P, Moguelet P, Khosrotehrani K, Aractingi S: Fetal microchimeric cells participate in tumour angiogenesis in melanomas occurring during pregnancy. Am J Pathol 2009, 174:630-637

13. Dubernard G, Oster M, Chareyre F, Antoine M, Rouzier R, Uzan S, Aractingi S, Khosrotehrani K: Increased fetal cell microchimerism in high grade breast carcinomas occurring during pregnancy. Int $\mathrm{J}$ Cancer 2009, 124:1054-1059

14. Dubernard G, Aractingi S, Oster M, Rouzier R, Mathieu MC, Uzan S, Khosrotehrani K: Breast cancer stroma frequently recruits fetal derived cells during pregnancy. Breast Cancer Res 2008, 10:R14

15. Nguyen Huu S, Oster M, Uzan S, Chareyre F, Aractingi S, Khosrotehrani K: Maternal neoangiogenesis during pregnancy partly derives from fetal endothelial progenitor cells. Proc Natl Acad Sci U S A 2007, 104:1871-1876

16. Zhang N, Fang Z, Contag PR, Purchio AF, West DB: Tracking angiogenesis induced by skin wounding and contact hypersensitivity using a Vegfr2-luciferase transgenic mouse. Blood 2004, 103:617-626

17. Lange-Asschenfeldt B, Weninger W, Velasco P, Kyriakides TR, von Andrian $\mathrm{UH}$, Bornstein P. Detmar M: Increased and prolonged inflammation and angiogenesis in delayed-type hypersensitivity reactions elicited in the skin of thrombospondin-2-deficient mice. Blood 2002, 99:538-545

18. Thijssen VL, Brandwijk RJ, Dings RP, Griffioen AW: Angiogenesis gene expression profiling in xenograft models to study cellular interactions. Exp Cell Res 2004, 299:286-293
19. Chakraborty AK, Yamaga S: Differential gene expression in genetically matched mouse melanoma cells with different metastatic potential. Gene 2003, 315:165-175

20. Gershenwald JE, Thompson W, Mansfield PF, Lee JE, Colome MI, Tseng CH, Lee JJ, Balch CM, Reintgen DS, Ross MI: Multi-institutional melanoma lymphatic mapping experience: the prognostic value of sentinel lymph node status in 612 stage I or II melanoma patients. J Clin Oncol 1999, 17:976-983

21. Hirakawa S, Kodama S, Kunstfeld R, Kajiya K, Brown LF, Detmar M: VEGF-A induces tumor and sentinel lymph node lymphangiogenesis and promotes lymphatic metastasis. J Exp Med 2005, 201:1089-1099

22. Harrell MI, Iritani BM, Ruddell A: Tumor-induced sentinel lymph node lymphangiogenesis and increased lymph flow precede melanoma metastasis. Am J Pathol 2007, 170:774-786

23. Massi D, Puig S, Franchi A, Malvehy J, Vidal-Sicart S, Gonzalez-Cao M, Baroni G, Ketabchi S, Palou J, Santucci M: Tumour lymphangiogenesis is a possible predictor of sentinel lymph node status in cutaneous melanoma: a case-control study. J Clin Pathol 2006, 59: $166-173$

24. Niakosari F, Kahn HJ, McCready D, Ghazarian D, Rotstein LE, Marks A, Kiss A, From L: Lymphatic invasion identified by monoclonal antibody D2-40, younger age, and ulceration: predictors of sentinel lymph node involvement in primary cutaneous melanoma. Arch Dermatol 2008, 144:462-467

25. Dadras SS, Lange-Asschenfeldt B, Velasco P, Nguyen L, Vora A, Muzikansky A, Jahnke K, Hauschild A, Hirakawa S, Mihm MC, Detmar $\mathrm{M}$ : Tumor lymphangiogenesis predicts melanoma metastasis to sentinel lymph nodes. Mod Pathol 2005, 18:1232-1242

26. Dadras SS, Paul T, Bertoncini J, Brown LF, Muzikansky A, Jackson DG, Ellwanger U, Garbe C, Mihm MC, Detmar M: Tumor lymphangiogenesis: a novel prognostic indicator for cutaneous melanoma metastasis and survival. Am J Pathol 2003, 162:1951-1960

27. Nathanson SD, Haas GP, Mead MJ, Lee M: Spontaneous regional lymph node metastases of three variants of the B16 melanoma: relationship to primary tumor size and pulmonary metastases. J Surg Oncol 1986, 33:41-45

28. Berston ED, Ramos DM, Kramer RH: Metastatic melanoma cells interact with the reticular fibres of the lymph node. Melanoma Res 1994, 4:115-125

29. Alterman AL, Fornabaio DM, Stackpole CW: Metastatic dissemination of B16 melanoma: pattern and sequence of metastasis. J Natl Cancer Inst 1985, 75:691-702

30. Larue L, Beermann F: Cutaneous melanoma in genetically modified animals. Pigment Cell Res 2007, 20:485-497

31. Isaka N, Padera TP, Hagendoorn J, Fukumura D, Jain RK: Peritumor lymphatics induced by vascular endothelial growth factor-C exhibit abnormal function. Cancer Res 2004, 64:4400-4404

32. Skobe M, Hawighorst $T$, Jackson DG, Prevo R, Janes L, Velasco $P$, Riccardi L, Alitalo K, Claffey K, Detmar M: Induction of tumor lymphangiogenesis by VEGF-C promotes breast cancer metastasis. Nat Med 2001, 7:192-198

33. Skobe M, Hamberg LM, Hawighorst T, Schirner M, Wolf GL, Alitalo K, Detmar M: Concurrent induction of lymphangiogenesis, angiogenesis, and macrophage recruitment by vascular endothelial growth factor-C in melanoma. Am J Pathol 2001, 159:893-903

34. Marcellini M, De Luca N, Riccioni T, Ciucci A, Orecchia A, Lacal PM, Ruffini F, Pesce M, Cianfarani F, Zambruno G, Orlandi A, Failla CM: Increased melanoma growth and metastasis spreading in mice overexpressing placenta growth factor. Am J Pathol 2006, 169:643-654

35. Shin JW, Huggenberger R, Detmar M: Transcriptional profiling of VEGF-A and VEGF-C target genes in lymphatic endothelium reveals endothelial-specific molecule-1 as a novel mediator of lymphangiogenesis. Blood 2008, 112:2318-2326

36. Slingluff CL Jr, Reintgen DS, Vollmer RT, Seigler HF: Malignant melanoma arising during pregnancy: a study of 100 patients. Ann Surg 1990, 211:552-557 